\title{
A note on the convex structure of uncertainty measures on MV-algebras
}

Tommaso Flaminio ${ }^{1}$ and Lluís Godo ${ }^{1}$

\begin{abstract}
In this paper we address the issue of providing a geometrical characterization for the decision problem of asking whether a partial assignment $\beta: f_{i} \mapsto \alpha_{i}$ mapping fuzzy events $f_{i}$ into real numbers $\alpha_{i}(i=1, \ldots, n)$ extends to a generalized belief function on fuzzy sets, according to a suitable definition. We will characterize this problem in a way that allows to treat it as the membership problem of a point to a specific convex set.
\end{abstract}

\section{Introduction}

The problem of deciding whether a partial assignment $v: f_{i} \mapsto \alpha_{i}$ mapping each event $f_{i}$ into a real number $\alpha_{i}$ (for $i=1, \ldots, s$ ) extends to a probability measure, is well known in the literature, and it is closely related with de Finetti's no-Dutch Book coherence criterion [3]. This criterion can be generalized in mainly two ways: by moving from classical to non-classical events (cf. $[14,16]$ for instance), or framing the problem out of the probabilistic setting, by taking into account alternative theories of uncertainty.

In $[14,16]$ the authors extend de Finetti's criterion for finitely-additive measures on non-classical events, and in particular in the case of those fuzzy events being representable as formulas of Lukasiewicz calculus. Following the proof of de Finetti's no-Dutch Book theorem (cf. [16, Theorem 2]), in $[14,16]$ extendible (i.e. coherent in de Finetti's terminology) assignments were characterized as Euclidean convex subsets of the finite dimensional space $\mathbb{R}^{s}$, $s$ being the number of events the assignment is defined over.

A similar approach to extendible assignments, but framed into an idempotent, rather than additive, setting has been developed in [8], where it is presented a geometrical characterization for the extendability problem for

IIIA Artificial Intelligence Research Institute, CSIC Spanish National Research Council. Campus UAB s/n, Bellaterra 08193, Spain, \{tommaso, godo\}@iiia.csic.es 
events being represented as normalized fuzzy sets over a finite domain in the context of possibility theory. It is worth recalling that in the frame of possibility theory, extendible assignments are again characterized as convex sets, but the Euclidean geometry has to be replaced by the min-plus geometry (see for instance [4]).

Belief functions are the measures used within Dempster-Shafer evidence theory [17] to quantify the amount of uncertainty associated to events. Among all the classical theories of uncertainty, Dempster-Shafer plays a pivotal role since both probability theory, and possibility theory can be obtained as particular cases. Belief functions have been recently extended to the MValgebraic setting to cope with spaces of events that can be organized as $\mathrm{MV}$-algebras of fuzzy subsets of a finite domain $X=\left\{x_{1}, \ldots, x_{n}\right\}[7,11]$.

In this paper we will provide a geometrical characterization for the extendability problem for events being (not necessarily normalized) fuzzy sets over a finite domain in a generalized framework of belief function theory. In particular, we will show how a mixture of min-plus convex geometry and Euclidean convex geometry can be applied to translate the extendability problem for belief functions, into the membership problem of a point to a convex set.

\section{Preliminaries}

In this section we will introduce the necessary preliminaries about the minplus convex geometry and MV-algebras. We will assume the reader to be familiar with Euclidean convex geometry, reminding that in what follows, given any subset $S$ of $\mathbb{R}^{s}, \operatorname{co}(S)$, and $\overline{\mathrm{co}}(S)$ will respectively stand for the convex hull of $S$ and its topological closure (with respect to the Euclidean metric). We invite the reader to consult [4] and [5] for all the unexplained notions.

\subsection{Preliminaries on min-plus convexity}

Let $\mathbf{x}_{1}, \ldots, \mathbf{x}_{n} \in \mathbb{R}^{s}$, and for every $i=1, \ldots, n$ and for every $t=1, \ldots, s$, let us denote by $\mathbf{x}_{i}(t)$ the $t$-th projection of $\mathbf{x}_{i}$. We then define the min-plus convex hull generated by $\mathbf{x}_{1}, \ldots, \mathbf{x}_{n}$ as the set

$$
\operatorname{mp}-\operatorname{co}\left(\mathbf{x}_{1}, \ldots, \mathbf{x}_{n}\right)=\left\{\mathbf{y} \in \mathbb{R}^{s}: \exists \lambda_{1}, \ldots, \lambda_{n} \in \mathbb{R}, \mathbf{y}(t)=\min _{i \leq n}\left(\lambda_{i}+\mathbf{x}_{i}(t)\right)\right\} .
$$

In the particular case of $\mathbf{x}_{1}, \ldots, \mathbf{x}_{n} \in[0,1]^{s}$, and $\lambda_{1}, \ldots, \lambda_{n} \in[0,1]$, we call bounded the combination $\min _{i \leq n}\left(\lambda_{i} \oplus \mathbf{x}_{i}\right)$. Notice that in the combination we replaced the usual sum with the bounded sum $x \oplus y=\min \{1, x+y\}$ to keep the combination in $[0,1]^{s}$ as well. More precisely, for $\mathbf{x}_{1}, \ldots, \mathbf{x}_{n} \in[0,1]^{s}$, we 
define the bounded min-plus convex hull generated by $\mathbf{x}_{1}, \ldots, \mathbf{x}_{n}$ as the set

$\operatorname{bmp}-\operatorname{co}\left(\mathbf{x}_{1}, \ldots, \mathbf{x}_{n}\right)=\left\{\mathbf{y} \in[0,1]^{s}: \exists \lambda_{1}, \ldots, \lambda_{n} \in[0,1], \mathbf{y}(t)=\min _{i \leq n}\left(\lambda_{i} \oplus \mathbf{x}_{i}(t)\right)\right\}$.

A bounded min-plus convex combination $\min _{i \leq n}\left(\lambda_{i} \oplus \mathbf{x}_{i}\right)$ is said to be normalized, if the parameters $\lambda_{1}, \ldots, \lambda_{n}$ satisfy $\max _{i \leq n} \lambda_{i}=1$, and therefore a bounded min-plus convex hull is said to be normalized accordingly. We will denote by nmp-co $(S)$ the normalized bounded min-plus convex hull generated by a set $S$.

\subsection{MV-algebras of fuzzy sets}

An $M V$-algebra $[1,15]$ is an algebra $(A, \oplus, \neg, \perp, \top)$ of type $(2,1,0,0)$ such that its reduct $(A, \oplus, \perp)$ is an abelian monoid, and the following equations hold for every $a, b \in A: \neg \neg a=a, a \oplus \top=\top$, and $\neg(\neg a \oplus b) \oplus b=\neg(\neg b \oplus a) \oplus a$.

Let $A$ and $B$ be MV-algebras. A $M V$-homomorphism is a map $h: A \rightarrow B$ sending $\perp$ and $\top$ of $A$ in $\perp$ and $\top$ of $B$ respectively, and such that, for every $a, a^{\prime} \in A, h\left(a \oplus a^{\prime}\right)=h(a) \oplus h\left(a^{\prime}\right)$, and $h(\neg a)=\neg h(a)$. We will denote by $\mathfrak{H}(A, B)$ the class of homomorphisms between $A$ and $B$.

Let $X=\left\{x_{1}, \ldots, x_{n}\right\}$ be a finite set of cardinality $n$, and consider the class $[0,1]^{X}$ of all fuzzy subsets of $X$, i.e. all functions from $X$ into the real unit interval $[0,1]$. This set can obviously be identified with the direct product $[0,1]^{n} .{ }^{1}$ The algebra obtained endowing $[0,1]^{n}$ with the point-wise operations $x \oplus y=\min \{1, x+y\}, \neg x=1-x$, and the two functions constantly equal to 0 and 1 , also denoted $\perp$ and $T$ respectively, is the typical example of MValgebra that we will consider in this paper as usual domain for uncertainty measures.

We will henceforth denote by $[0,1]^{n}$ both the domain, and the MV-algebra above defined without danger of confusion, moreover, we will always assume $X$ to be finite. Notice that, whenever $X$ consists of just one element, $[0,1]^{1}$ is the (linearly ordered) MV-algebra over the real unit interval. This algebra, that is usually named the standard MV-algebra, will be denoted by $[0,1]_{M V}$.

For every $f=\langle f(1), \ldots, f(n)\rangle \in[0,1]^{n}$, we will henceforth consider the function $\rho_{f}:[0,1]^{n} \rightarrow[0,1]$ mapping every $b \in[0,1]^{n}$ into

$$
\rho_{f}(b)=\min _{i \leq n}(\neg b(i) \oplus f(i)),
$$

Those mappings $\rho_{f}$ can be regarded as generalized inclusion operators between fuzzy sets (cf. [7] for further details). For every $f \in\{0,1\}^{n}$ (i.e.

1 The set $X=\left\{x_{1}, \ldots, x_{n}\right\}$ can be equivalently identified with the set of its indices $\{1, \ldots, n\}$. This allows in turn to identify each function $f \in[0,1]^{X}$ as a point $\langle f(1), \ldots, f(n)\rangle \in[0,1]^{n}$ and vice-versa. In what follows we will equivalently use the notation $[0,1]^{n}$ or $[0,1]^{X}$ for the MV-algebra of $[0,1]$-valued functions over $X$. 
whenever $f$ is identified with a vector in $[0,1]^{n}$ with integer components), the map $\rho_{f}:[0,1]^{n} \rightarrow[0,1]$ is a pointwise minimum of finitely many linear functions with integer coefficients, and hence $\rho_{f}$ is a non-increasing McNaughton function $[1,15]$. Letting $\mathcal{R}$ to be the MV-algebra generated by the set $\left\{\rho_{f}: f \in[0,1]^{n}\right\},[1$, Theorem 3.4.3] and [13, Theorem 2.5], allow to prove the following result.

Theorem 1. There exists a one-to-one correspondence between the points of $[0,1]^{n}$ and the class $\mathfrak{H}\left(\mathcal{R},[0,1]_{M V}\right)$ of homomorphisms of $\mathcal{R}$ into the standard $M V$-algebra $[0,1]_{M V}$.

Thanks to the above Theorem 1 we will henceforth identify points in $[0,1]^{n}$ (hence fuzzy subsets of $X$ ) with homomorphisms of $\mathcal{R}$ in the MV-algebra $[0,1]_{M V}$ without loss of generality. Moreover, the following holds:

Corollary 1. Let $\left\{\tau_{1}, \ldots, \tau_{s}\right\}$ be a finite subset of $\mathcal{R}$. Then $\left\{\left\langle h\left(\tau_{1}\right), \ldots, h\left(\tau_{s}\right)\right\rangle \in\right.$ $\left.[0,1]^{s}: h \in \mathfrak{H}\left(\mathcal{R},[0,1]_{M V}\right)\right\}=\left\{\left\langle\tau_{1}(a), \ldots, \tau_{s}(a)\right\rangle: a \in[0,1]^{n}\right\}$.

\section{Uncertainty measures on MV-algebras of fuzzy sets}

In this section we are going to recall how states [13] and necessity measures [6], can be defined on MV-algebras (of fuzzy sets). In particular we will also recall and prove how the problem of extending a partial assessment can be geometrically characterized in these frameworks.

A state on an MV-algebra $A$ is a map $s: A \rightarrow[0,1]$ satisfying: (1) $s(\top)=1$; (2) for every $a, b \in A$ such that $\neg(\neg a \oplus \neg b)=\perp, s(a \oplus b)=s(a)+s(b)$. A state $s$ is said to be faithful provided that $s(a)=0$, implies $a=\perp$.

States play the same role on MV-algebras as probability measures do on Boolean algebras. In particular the well known de Finetti's extension theorem was generalized to the case of states and MV-algebras by Mundici [14]. Below we recall it in the particular case of the MV-algebra being $[0,1]^{X}$.

Theorem 2. Let $f_{1}, \ldots, f_{s}$ be elements in $[0,1]^{X}$. Then a map $\sigma: f_{i} \mapsto \alpha_{i} \in$ $[0,1]$ extends to a state on $[0,1]^{X}$ iff $\left\langle\alpha_{1}, \ldots, \alpha_{s}\right\rangle \in \overline{\mathrm{co}}\left\{\left\langle h\left(f_{1}\right), \ldots, h\left(f_{s}\right)\right\rangle\right.$ : $\left.h \in \mathfrak{H}\left([0,1]^{X},[0,1]_{M V}\right)\right\}$.

As in Theorem 1 , the class $\mathfrak{H}\left([0,1]^{X},[0,1]_{M V}\right)$ is in one-to-one correspondence with the set $X=\left\{x_{1}, \ldots, x_{n}\right\}$ and hence from Theorem 2 an assignment $\sigma$ : $f_{i} \mapsto \alpha_{i}$ extends to a state on $[0,1]^{X}$ iff $\left\langle\alpha_{1}, \ldots, \alpha_{s}\right\rangle \in \operatorname{co}\left\{\left\langle f_{1}(x), \ldots, f_{s}(x)\right\rangle\right.$ : $x \in X\rangle\}^{2}$. The result is shown in Fig. 1 .

Necessity measures on MV-algebras like $[0,1]^{X}$ have been introduced in [6]: a map $N:[0,1]^{X} \rightarrow[0,1]$ is a necessity measure, provided that $N(\top)=1$,

${ }^{2}$ Notice that since $X$ is finite, so is $F=\left\{\left\langle f_{1}(x), \ldots, f_{s}(x): x \in X\right\rangle\right\}$, and hence its convex hull is the polytope generated by $F$ which is already closed. In other words, $\overline{\mathrm{co}}(F)=\operatorname{co}(F)$. 


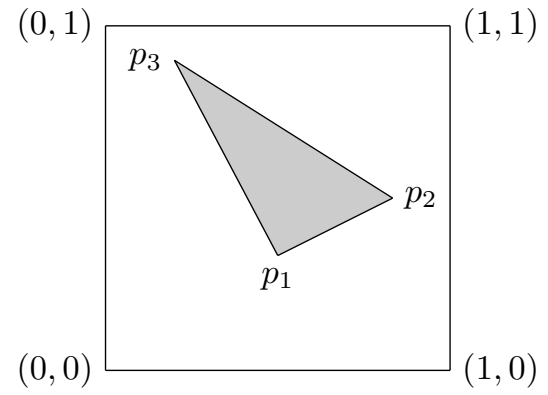

Fig. 1 Let $X=\{1,2,3\}$, consider two elements $f_{1}, f_{2} \in[0,1]^{3}$, and the assignment $\sigma\left(f_{1}\right)=\alpha_{1}$, and $\sigma\left(f_{2}\right)=\alpha_{2}$. The two functions $f_{1}, f_{2}: X \rightarrow[0,1]$ defines three points in the unit square $[0,1]^{2}$, namely:

$p_{1}=\left(f_{1}(1), f_{2}(1)\right)$

$p_{2}=\left(f_{1}(2), f_{2}(2)\right)$

$p_{3}=\left(f_{1}(3), f_{2}(3)\right)$.

Therefore the assignment $\sigma$ extends to a state on $[0,1]^{3}$ iff the point $\left\langle\alpha_{1}, \alpha_{2}\right\rangle$ belongs to the triangle with vertices $p_{1}, p_{2}$, and $p_{3}$.

for every $f, f^{\prime} \in[0,1]^{X}, N\left(f \wedge f^{\prime}\right)=\min \left\{N(f), N\left(f^{\prime}\right)\right\}$, and for every $f, \bar{r} \in$ $[0,1]^{X}$, if $\bar{r}$ is the function constantly equal to $r$, then $N(\bar{r} \oplus f)=r \oplus N(f){ }^{3}$

Actually necessity measures on $[0,1]^{X}$ can be equivalently defined as follows: if $\pi: X \rightarrow[0,1]$ is a map called a possibility distribution, then we define $N_{\pi}: f \in[0,1]^{X} \mapsto \min _{x \in X}\{(1-\pi(x)) \oplus f(x)\} \in[0,1]$, and in this case we say that $N_{\pi}$ is defined by $\pi$. Conversely in [6, Theorem 3.3] it is shown that for every necessity measure $N$ on $[0,1]^{X}$, there exists a (unique) possibility distribution $\pi$ defining $N$.

A necessity measure $N$ is said to be normalized provided that the possibility distribution $\pi$ defining $N$ satisfies $\max _{x \in X} \pi(x)=1$, and is called nonnormalized otherwise. Notice that, if $N$ is not normalized, then $N(\perp)>0$. On the other hand, normalized necessities always satisfy $N(\perp)=0$. We will denote by $\mathcal{N}\left([0,1]^{X}\right)$ the class of necessity measures on $[0,1]^{X}$. Normalization will always be clear by the context.

The following can be proved going through the lines of [8, Theorem 4].

Theorem 3. Let $f_{1}, \ldots, f_{s} \in[0,1]^{X}$, and let $\eta: f_{i} \mapsto \alpha_{i}$ be an assessment. Then the following hold:

1. $\eta$ extends to a non-normalized necessity measure iff

$$
\left\langle\alpha_{1}, \ldots, \alpha_{s}\right\rangle \in \operatorname{bmp}-\operatorname{co}\left(\left\{\left\langle f_{1}\left(x_{i}\right), \ldots, f_{s}\left(x_{i}\right)\right\rangle: 1 \leq i \leq n\right\}\right) .
$$

2. $\eta$ extends to a normalized necessity measure iff

$$
\left\langle\alpha_{1}, \ldots, \alpha_{s}\right\rangle \in \operatorname{nmp}-\operatorname{co}\left(\left\{\left\langle f_{1}\left(x_{i}\right), \ldots, f_{s}\left(x_{i}\right)\right\rangle: 1 \leq i \leq n\right\}\right) .
$$

Example 1. Let $X=\{1,2,3\}$, consider two elements $f_{1}, f_{2} \in[0,1]^{3}$, and an assessment $\sigma\left(f_{1}\right)=\alpha_{1}$, and $\sigma\left(f_{2}\right)=\alpha_{2}$. The two functions $f_{1}, f_{2}: X \rightarrow[0,1]$ define three points in the unit square $[0,1]^{2}$, namely: $p_{1}=\left(f_{1}(1), f_{2}(1)\right) ; p_{2}=$

\footnotetext{
${ }^{3}$ In $[6,8]$ necessity measures also satisfying the last condition on constant functions were called homogeneous necessity measures. In this paper, since we will not distinguish between homogeneous and non-homogeneous mappings, we will use to call them necessity measures without danger of confusion.
} 

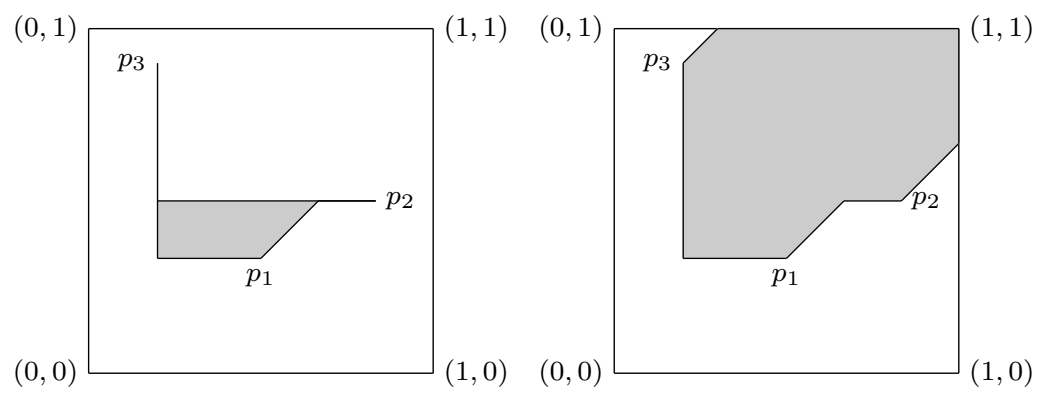

Fig. 2 Three points in the unit square $[0,1]^{2}, p_{1}, p_{2}$ and $p_{3}$, and their normalized (left) and non-normalized (right) min-plus convex hull.

$\left(f_{1}(2), f_{2}(2)\right) ; p_{3}=\left(f_{1}(3), f_{2}(3)\right)$. Therefore the assignment $\sigma$ respectively extends to a normalized (resp. non-normalized) necessity measure on $[0,1]^{3}$ iff the point $\left\langle\alpha_{1}, \alpha_{2}\right\rangle$ belongs to the normalized (resp. non-normalized) minplus convex polygon of vertices $p_{1}, p_{2}$, and $p_{3}$, depicted respectively in Figure 2 on the left and on the right.

Next result shows that the inclusion operators $\rho_{(\cdot)}(b)$, with varying $b \in$ $[0,1]^{X}$, generate the whole class of necessity measures on $[0,1]^{X}$, and will be useful in the next section.

Lemma 1. (1) The class of all necessity measures on $[0,1]^{X}$ coincides with the class $\left\{\rho_{(\cdot)}(b): f \in[0,1]^{X} \mapsto \rho_{f}(b) \mid b \in[0,1]^{X}\right\}$.

(2) The class of all normalized necessity measures on $[0,1]^{X}$ coincides with the class $\left\{\rho_{(\cdot)}(b): f \in[0,1]^{X} \mapsto \rho_{f}(b) \mid b \in[0,1]^{X}, \max _{x \in X} b(x)=1\right\}$.

Corollary 2. Let $f_{1}, \ldots, f_{s} \in[0,1]^{X}$, and let $\eta: f_{i} \mapsto \alpha_{i}$ be an assessment. Then:

1. $\eta$ extends to a non-normalized necessity in $\mathcal{N}\left([0,1]^{X}\right)$ iff

$$
\left\langle\alpha_{1}, \ldots, \alpha_{s}\right\rangle \in\left\{\left\langle\rho_{f_{1}}(b), \ldots, \rho_{f_{s}}(b)\right\rangle: b \in[0,1]^{X}\right\} .
$$

2. $\eta$ extends to a normalized necessity in $\mathcal{N}\left([0,1]^{X}\right)$ iff

$$
\left\langle\alpha_{1}, \ldots, \alpha_{s}\right\rangle \in\left\{\left\langle\rho_{f_{1}}(b), \ldots, \rho_{f_{s}}(b)\right\rangle: b \in[0,1]^{X}, \max _{x \in X} b(x)=1\right\} .
$$

\section{Belief functions and the extendability problem}

Let us recall from Subsection 2.2 that, for every MV-algebra $[0,1]^{X}$, the algebra $\mathcal{R}$ is defined as the MV-algebra generated by all functions $\rho_{f}$ defined as in (1). Then belief functions on $[0,1]^{X}$ can be defined along the proposals in $[7,11,12]$. 
Definition 1. A map Bel $:[0,1]^{X} \rightarrow[0,1]$ is a belief function if there exists a state $s: \mathcal{R} \rightarrow[0,1]$ such that for every $a \in[0,1]^{X}, \operatorname{Bel}(a)=s\left(\rho_{a}\right)$.

Notice that, in general, belief functions on $[0,1]^{X}$ fail to satisfy $\operatorname{Bel}(\perp)=0$. In fact, for every $b \in[0,1]^{X}$ such that $\max _{x \in X} b(x)<1, \rho_{\perp}(b)>0$, and hence $\rho_{\perp}$ does not coincide with the function constantly equal to 0 . Therefore, whenever $s$ is faithful, $\operatorname{Bel}(\perp)=s\left(\rho_{\perp}\right)>0$. We will henceforth call normalized any belief function satisfying $\operatorname{Bel}(\perp)=0$.

The following result provides a geometrical characterization for the extendability problem for belief functions on MV-algebras.

Theorem 4. Let $f_{1}, \ldots, f_{s} \in[0,1]^{X}$, and let $\beta: f_{i} \mapsto \alpha_{i}$ be $a[0,1]$-valued mapping. Then the following hold:

1. $\beta$ extends to a belief function $\mathrm{Bel}$ on $[0,1]^{X}$ iff

$$
\left\langle\alpha_{1}, \ldots, \alpha_{s}\right\rangle \in \overline{\mathrm{co}}\left(\operatorname{bmp}-\operatorname{co}\left(\left\{\left\langle f_{1}\left(x_{i}\right), \ldots, f_{s}\left(x_{i}\right)\right\rangle: i \leq n\right\}\right)\right) .
$$

2. $\beta$ extends to a normalized belief function Bel on $[0,1]^{n}$ iff

$$
\left\langle\alpha_{1}, \ldots, \alpha_{s}\right\rangle \in \overline{\mathrm{co}}\left(\mathrm{nmp}-\operatorname{co}\left(\left\{\left\langle f_{1}\left(x_{i}\right), \ldots, f_{s}\left(x_{i}\right)\right\rangle: i \leq n\right\}\right)\right) .
$$

Proof. 1. The assignment $\beta$ extends to a belief function $\mathrm{Bel}:[0,1]^{X} \rightarrow$ $[0,1]$ iff there exists a state $s: \mathcal{R} \rightarrow[0,1]$ such that, for every $t=1, \ldots, s$, $\alpha_{t}=s\left(\rho_{f_{t}}\right)=\operatorname{Bel}\left(f_{t}\right)$. From Theorem 2, this means that $\left\langle\alpha_{1}, \ldots, \alpha_{s}\right\rangle \in$ $\overline{\mathrm{co}}\left\{\left\langle h\left(\rho_{f_{1}}\right), \ldots, h\left(\rho_{f_{s}}\right)\right\rangle \in[0,1]^{s}: h \in \mathfrak{H}\left(\mathcal{R},[0,1]_{M V}\right)\right\}$. From Corollary 1, (??) is equivalent to: $\left\langle\alpha_{1}, \ldots, \alpha_{s}\right\rangle \in \overline{\operatorname{co}}\left\{\left\langle\rho_{f_{1}}(b), \ldots, \rho_{f_{s}}(b)\right\rangle \in[0,1]^{s}: b \in\right.$ $\left.[0,1]^{X}\right\}$. From (1) of Corollary 2, the set $\left\{\left\langle\rho_{f_{1}}(b), \ldots, \rho_{f_{s}}(b)\right\rangle \in[0,1]^{s}: b \in\right.$ $\left.[0,1]^{X}\right\}$ coincides with the set of all the coherent necessity assignments over $\left\{f_{1}, \ldots, f_{s}\right\}$, in other words $y \in\left\{\left\langle\rho_{f_{1}}(b), \ldots, \rho_{f_{s}}(b)\right\rangle \in[0,1]^{s}: b \in[0,1]^{X}\right\}$ iff there exists a necessity measure $N$ such that for every $t, N\left(f_{t}\right)=y(t)$. Finally Theorem 3 implies that the set of all coherent necessity assessments over $f_{1}, \ldots, f_{s}$ coincides with bmp-co $\left(\left\{\left\langle f_{1}\left(x_{i}\right), \ldots, f_{s}\left(x_{i}\right)\right\rangle: i \leq n\right\}\right)$, and hence our the claim is settled.

2. The proof runs completely parallel to the previous one and is omitted.

Example 2. (Example 1 continued) Let $X=\{1,2,3\}$, consider two elements $f_{1}, f_{2} \in[0,1]^{3}$, and an assessment $\sigma\left(f_{1}\right)=\alpha_{1}$, and $\sigma\left(f_{2}\right)=\alpha_{2}$. Therefore the assignment $\sigma$ extends either to a normalized belief function, or to a belief function in general, on $[0,1]^{3}$ iff the point $\left\langle\alpha_{1}, \alpha_{2}\right\rangle$ belongs respectively to the convex polytope on the left in Figure 3, or to the convex polytope on the right of the same Figure 3.

Acknowledgements. The authors acknowledge partial support from the Spanish projects TASSAT (TIN2010- 20967-C04-01), Agreement Technologies (CONSOLIDER CSD2007-0022, INGENIO 2010) and ARINF (TIN2009-14704-C03-03). Flaminio acknowledges partial support from the Juan de la Cierva Program of the Spanish MICINN. 

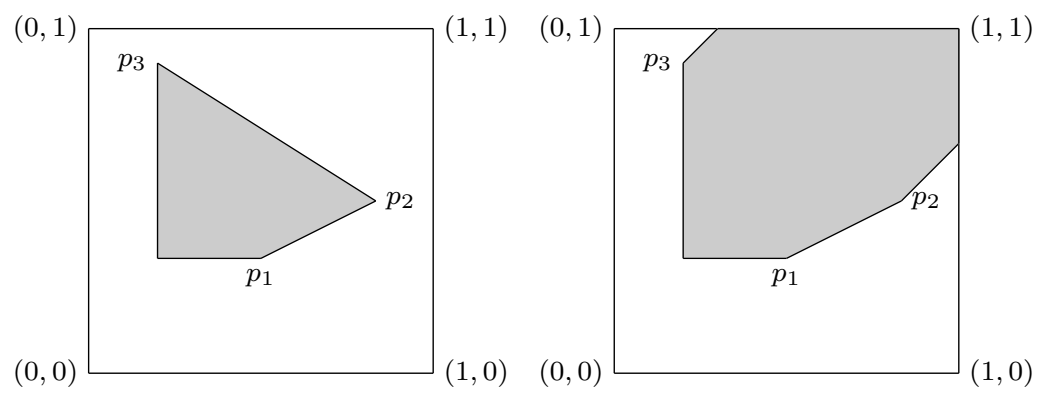

Fig. 3 Two convex polytopes in the unit square $[0,1]^{2}$ defined by vertices $p_{1}, p_{2}$ and $p_{3}$.

\section{References}

1. R. Cignoli, I.M.L. D'Ottaviano, D. Mundici, Algebraic Foundations of Many-valued Reasoning. Kluwer, Dordrecht, 2000.

2. P.M. Cohn. Universal Algebra. Revisited Edition, D. Reidel Pub. Co., Dordrecht, 1981.

3. B. de Finetti, Theory of Probability, Vol.1, Wiley, New York, 1974.

4. M. Develin, B. Sturmfels. Tropical convexity. Doc. Math. 9:1-27, 2004.

5. G. Edwald. Combinatorial Convexity and Algebraic Geometry. Springer, New York, 1996.

6. T. Flaminio, L. Godo, E. Marchioni. On the logical formalization of possibilistic counterpart of states over n-valued Łukasiewicz events. J. Logic Comput. 21(3):447-464, 2011.

7. T. Flaminio, L. Godo, E. Marchioni. Belief Functions on MV-Algebras of Fuzzy Events Based on Fuzzy Evidence. In Proceedings of ECSQARU 2011. Lecture Notes in Artificial Intelligence 6717, Weiru Liu (Ed.):628-639, 2011.

8. T. Flaminio, L. Godo, E. Marchioni. Geometrical aspects of possibility measures on finite domain MV-clans. Soft Computing. DOI 10.1007/s00500-012-0838-0.

9. K. R. Goodearl, Partially Ordered Abelian Group with Interpolation. AMS Math. Survey and Monographs, vol 20, 1986.

10. T. Kroupa, Every state on semisimple MV-algebra is integral. Fuzzy Sets and Systems, 157:2771-2782, 2006.

11. T. Kroupa, From Probabilities to Belief Functions on MV-algebras, in Combining Soft Computing and Statistical Methods in Data Analysis, volume 77 of Advances in Soft Computing:387-394. Springer-Verlag, 2010.

12. T. Kroupa, Extension of Belief Functions to Infinite-valued Events. Soft Computing, DOI 10.1007/s00500-012-0836-2, 2012.

13. D. Mundici, Averaging the truth-value in Łukasiewicz logic. Studia Logica, 55(1):113127,1995

14. D. Mundici, Bookmaking over infinite-valued events. Int. J. Approx. Reason. 43:223$240,2006$.

15. D. Mundici, Advanced Eukasiewicz calculus and MV-algebras. Trends in Logic, vol 35. Springer, 2011.

16. J. B. Paris. A note on the Dutch Book method, Revised version of a paper of the same title which appeared in The Proceedings of the Second Internat. Symp. on Imprecise Probabilities and their Applications, ISIPTA01, Ithaca, New York, 2001.

17. G. Shafer. A Mathematical Theory of Evidence. Princeton University Press, Princeton 1976. 


\section{Appendix}

\section{Theorem 1}

Proof. For every $n$ the product space $[0,1]^{n}$ is compact and Hausdorff, and hence $\mathcal{R}$ a MV-subalgebra of the MV-algebra of continuous function defined over a compact Hausdorff space. Moreover $\mathcal{R}$ is a separating, that is for every $x \neq y$ in $[0,1]^{n}$, there exists a function $f \in \mathbb{R}$, such that $f(x) \neq f(y)$. Therefore, from [1, Theorem 3.4.3] there exists a one-to-one correspondence between $[0,1]^{n}$ and the class $\mathcal{M}(\mathcal{R})$ of its maximal ideals.

Now, from [13, Theorem 2.5] (see also [9, Theorem 12.18, Corollary 12.20], and $\left[10\right.$, Lemma 23]), the class $\mathfrak{H}\left(\mathcal{R},[0,1]_{M V}\right)$ is in one-to-one correspondence with $\mathcal{M}(\mathcal{R})$, and hence the claim follows.

\section{Corollary 1}

Proof. For every $x \in[0,1]^{n}$ the map $h_{x}: \mathcal{R} \rightarrow[0,1]_{M V}$ such that for every $f \in \mathcal{R}, h_{x}(f)=f(x)$ is a homomorphism of $\mathcal{R}$ in $[0,1]_{M V}$. From [10, Theorem $25]$ the map $x \mapsto h_{x}$ is one-to-one. Then the claim easily follows.

\section{Lemma 1}

Proof. In [7, Proposition 1] it is shown that for every $a, b \in[0,1]^{n}, \rho_{a \wedge b}(f)=$ $\rho_{a}(f) \wedge \rho_{b}(f)$. Moreover, for every $a, b, f, r \in[0,1]^{n}$, it is easy to check that $\rho_{\top}(f)=1$, and $\rho_{r \oplus a}(f)=r \oplus \rho_{a}(f)$ whenever $r$ is constant. Hence, for every $f \in[0,1]^{n}, \rho_{(\cdot)}(f) \in \mathcal{N}\left([0,1]^{n}\right)$.

As for the other inclusion let us identify every (normalized) possibility distribution $\pi:\{1, \ldots, n\} \rightarrow[0,1]$, with a point in $f_{\pi} \in[0,1]^{n}$. Then, for every $a \in[0,1]^{n}$,

$$
N_{\pi}(a)=\min _{i \leq n} \neg \pi(i) \oplus a(i)=\min _{i \leq n} \neg f_{\pi}(i) \oplus a(i)=\rho_{a}\left(x_{\pi}\right) .
$$

Therefore $N_{\pi} \in\left\{\rho_{(\cdot)}(b): f \in[0,1]^{X} \mapsto \rho_{f}(b) \mid b \in[0,1]^{X}\right\}\left(N_{\pi} \in\left\{\rho_{(\cdot)}(b)\right.\right.$ : $\left.f \in[0,1]^{X} \mapsto \rho_{f}(b) \mid b \in[0,1]^{X}, \max _{x \in X} b(x)=1\right\}$ respectively).

\section{Corollary 2}

Proof. 1. $\left\langle\alpha_{1}, \ldots, \alpha_{s}\right\rangle \in\left\{\left\langle\rho_{f_{1}}(b), \ldots, \rho_{f_{s}}(b)\right\rangle: b \in[0,1]^{n}\right\}$ iff there exists a $b \in[0,1]^{n}$ such that $\left\langle\alpha_{1}, \ldots, \alpha_{s}\right\rangle=\left\langle\rho_{f_{1}}(b), \ldots, \rho_{f_{s}}(b)\right\rangle$, iff (from Lemma 1), $\left\langle\alpha_{1}, \ldots, \alpha_{s}\right\rangle=\left\langle N_{b}\left(f_{1}\right), \ldots, N_{b}\left(f_{s}\right)\right\rangle$ for some $N_{b} \in \mathcal{N}\left([0,1]^{n}\right)$. The case 2 . is similar and omitted. 